\title{
Endogenous erythropoietin at birth is associated with neurodevelopmental morbidity in early childhood
}

\author{
Elina J. Rancken $\mathbb{D}^{1 凶}{ }^{凶}$, Marjo P. H. Metsäranta ${ }^{1}$, Mika Gissler ${ }^{2,3}$, Leena K. Rahkonen ${ }^{4}$ and Leena M. Haataja ${ }^{1}$
}

(c) The Author(s) 2021

BACKGROUND: New biomarkers that predict later neurodevelopmental morbidity are needed. This study evaluated the associations between umbilical cord serum erythropoietin (us-EPO) and neurodevelopmental morbidity by the age of 2-6.5 years in a Finnish cohort.

METHODS: This study included 878 non-anomalous children born alive in 2012 to 2016 in Helsinki University Hospitals and whose us-EPO concentration was determined at birth. Data of these children were linked to data from the Finnish Medical Birth Register and the Finnish Hospital Discharge Register. Neurodevelopmental morbidity included cerebral palsy, epilepsy, intellectual disability, autism spectrum disorder, sensorineural defects, and minor neurodevelopmental disorders.

RESULTS: In the cohort including both term and preterm children, us-EPO levels correlated with gestational age $(r=0.526)$ and were lower in premature children. High us-EPO levels (>100 IU/I) were associated with an increased risk of severe

neurodevelopmental morbidity (OR: $4.87 ; 95 \% \mathrm{Cl}$ : 1.05-22.58) when adjusted for the gestational age. The distribution of us-EPO levels did not differ in children with or without the later neurodevelopmental diagnosis.

CONCLUSIONS: Although high us-EPO concentration at birth was associated with an increased risk of neurodevelopmental morbidity in early childhood, the role of us-EPO determination in clinical use appears to be minor.

Pediatric Research (2022) 92:307-314; https://doi.org/10.1038/s41390-021-01679-0

\section{IMPACT:}

- We determined whether endogenous umbilical cord serum erythropoietin would be a new useful biomarker to predict the risk of neurodevelopmental morbidity.

- This study evaluated the role of endogenous erythropoietin at birth in neurodevelopmental morbidity with a study population of good size and specific diagnoses based on data from high-quality registers.

- Although high umbilical cord serum erythropoietin concentration at birth was associated with an increased risk of neurodevelopmental morbidity in early childhood, the clinical value of erythropoietin determination appears to be minor.

\section{INTRODUCTION}

Human erythropoietin (EPO), a hormone whose synthesis increases under hypoxic conditions, regulates red blood cell production from the fetal stage. ${ }^{1}$ Elevated EPO levels in fetal plasma correlate well with the intensity of fetal hypoxia, ${ }^{2}$ as EPO does not pass through the placenta ${ }^{3}$ and is not stored in tissues.

According to preclinical studies, EPO has many neuroprotective effects. ${ }^{4}$ Preliminary results using EPO as a neuroprotective agent were promising in neonatal hypoxic-ischemic encephalopathy, ${ }^{5}$ and showed an improvement in cognitive outcome in very preterm infants. ${ }^{6,7}$ However, two randomized, placebo-controlled trials from 2016 and 2020 reported that, in extremely and very preterm infants, high-dose EPO treatment did not decrease the risk of neurological impairment at 2 years of age assessed using the Bayley Scales of Infant Development. ${ }^{8,9}$ In a further study in very preterm infants, the neurodevelopmental outcome showed no difference later at 5 years of age either. ${ }^{10}$

As for endogenous EPO, high endogenous EPO concentration in blood during the first postnatal days has been associated with neonatal morbidity in extremely preterm infants ${ }^{11}$ and with neonatal encephalopathy, abnormal MRI and mortality in term infants exposed to perinatal asphyxia. ${ }^{12}$ Minimal data exist on the association between endogenous EPO levels at birth and longterm neurodevelopmental morbidity in children. One follow-up study from 1988 reported that high fetal plasma EPO levels were associated with an increased risk of cerebral palsy or death at 2 years of age. ${ }^{13}$ Further, Korzeniewski et al. found that high EPO levels on a postnatal day 14 are associated with adverse outcomes (very low mental and psychomotor development indices and microcephaly) at 2 years of age in extremely preterm infants. ${ }^{14}$

\footnotetext{
${ }^{1}$ Children's Hospital, Pediatric Research Centre, Helsinki University Hospital, University of Helsinki, Helsinki, Finland. ${ }^{2}$ Information Services Department, Finnish Institute for Health and Welfare, Helsinki, Finland. ${ }^{3}$ Department of Neurobiology, Care Sciences and Society, Karolinska Institute, Stockholm, Sweden. ${ }^{4}$ Department of Obstetrics and Gynecology, Helsinki University Hospital, University of Helsinki, Helsinki, Finland. ${ }^{凶}$ email: elina.rancken@helsinki.fi
}

Received: 16 February 2021 Revised: 26 July 2021 Accepted: 27 July 2021

Published online: 31 August 2021 
Given the role of EPO in hypoxic-ischemic insult, we evaluated whether elevated umbilical cord serum EPO (us-EPO) levels at birth are associated with increased risks of severe neurodevelopmental morbidity by the age of 2-6.5 years in a Finnish cohort including both term and preterm children. Major neurodevelopmental morbidity included cerebral palsy, epilepsy, intellectual disability, autism spectrum disorder, and sensorineural defects consisting of visual and hearing impairments. The secondary aim was to assess the association of us-EPO with minor neurodevelopmental disorders in early childhood and infant mortality by the age of 1 year.

\section{PATIENTS AND METHODS}

\section{Population and data sources}

This register-based retrospective study included children born between 1 May 2012 and 31 December 2016 in maternity hospitals in Helsinki University Hospital and whose us-EPO concentration was determined from

Table 1. Characteristics of the study population, overall and stratified by us-EPO categories.

\begin{tabular}{|c|c|c|c|c|c|c|c|c|}
\hline \multirow[b]{3}{*}{ Characteristics } & \multirow{3}{*}{$\begin{array}{l}\text { Total } \\
\text { n (\%) }\end{array}$} & \multicolumn{7}{|c|}{ us-EPO level at birth } \\
\hline & & \multicolumn{2}{|l|}{ Low $<20$} & \multirow{2}{*}{$\begin{array}{l}\text { Average } 20-39 \\
n(\%) \\
\text { Reference group }\end{array}$} & \multicolumn{2}{|c|}{ Elevated 40-100 } & \multicolumn{2}{|l|}{ High > 100} \\
\hline & & $n(\%)$ & $p$-value ${ }^{a}$ & & $n(\%)$ & $p$-value ${ }^{a}$ & $n(\%)$ & $p$-value ${ }^{a}$ \\
\hline Total & $878(100.0)$ & $218(100.0)$ & & $218(100.0)$ & $215(100.0)$ & & $227(100.0)$ & \\
\hline \multicolumn{9}{|l|}{ Mode of delivery } \\
\hline Vaginal delivery & $329(37.5)$ & $107(49.1)$ & \multirow[t]{5}{*}{0.113} & $104(47.7)$ & $78(36.3)$ & \multirow[t]{5}{*}{0.103} & $40(17.6)$ & \multirow[t]{5}{*}{$<0.001$} \\
\hline Vacuum extraction/forceps & $193(22.0)$ & $45(20.6)$ & & $53(24.3)$ & $58(27.0)$ & & $37(16.3)$ & \\
\hline Planned cesarean section & $9(1.0)$ & $7(3.2)$ & & $0(0.0)$ & $1(0.5)$ & & $1(0.4)$ & \\
\hline Acute cesarean section & $220(25.1)$ & $29(13.3)$ & & $35(16.1)$ & $53(24.7)$ & & $103(45.4)$ & \\
\hline Emergency cesarean section & $107(12.2)$ & $26(11.9)$ & & $21(9.6)$ & $18(8.4)$ & & $42(18.5)$ & \\
\hline Male & $498(56.7)$ & $127(58.3)$ & 0.178 & $113(51.8)$ & $127(59.1)$ & 0.130 & $131(57.7)$ & 0.213 \\
\hline $32+0-36+6$ & $77(8.8)$ & $28(12.8)$ & \multirow{3}{*}{$<0.001$} & $13(6.0)$ & $7(3.3)$ & \multirow{3}{*}{0.117} & $29(12.8)$ & \multirow{3}{*}{0.004} \\
\hline $37+0-41+6$ & $676(77.0)$ & $169(77.5)$ & & $179(82.1)$ & $167(77.7)$ & & $161(70.9)$ & \\
\hline$\geq 42+0$ & $102(11.6)$ & $7(3.2)$ & & $23(10.6)$ & $38(17.7)$ & & $34(15.0)$ & \\
\hline Birth weight $(g$, mean $\pm S D)$ & 3387 (701) & 3224 (814) & $<0.001$ & $3504(610)$ & $3517(665)$ & 0.831 & 3307 (658) & 0.001 \\
\hline$<2500$ & $73(8.3)$ & $25(11.5)$ & 0.025 & $12(5.5)$ & $16(7.4)$ & 0.412 & $20(8.8)$ & 0.177 \\
\hline$\geq 4500$ & $20(2.3)$ & $3(1.4)$ & 0.475 & $5(2.3)$ & $6(2.8)$ & 0.742 & $6(2.6)$ & 0.812 \\
\hline \multicolumn{9}{|l|}{ Birth weight (g) } \\
\hline SGA & $60(6.8)$ & $10(4.6)$ & 0.401 & $14(6.4)$ & $10(4.7)$ & 0.421 & $26(11.5)$ & 0.064 \\
\hline LGA & $26(3.0)$ & $10(4.6)$ & 0.630 & $8(3.7)$ & $5(2.3)$ & 0.413 & $5(2.2)$ & 0.358 \\
\hline \multicolumn{9}{|l|}{ 1-min Apgar score } \\
\hline $7-10$ & $590(67.2)$ & $161(73.9)$ & \multirow{2}{*}{0.998} & $161(73.9)$ & $148(68.8)$ & \multirow{2}{*}{0.450} & $120(52.9)$ & \multirow{2}{*}{0.001} \\
\hline No information & $3(0.3)$ & $1(0.5)$ & & $1(0.5)$ & $0(0.0)$ & & $1(0.4)$ & \\
\hline Umbilical artery $\mathrm{pH}$ & & & & & & & & \\
\hline$<7.00$ & $75(8.5)$ & $12(5.5)$ & 0.314 & $19(8.7)$ & $24(11.2)$ & 0.426 & $20(8.8)$ & 0.570 \\
\hline $7.00-7.10$ & $144(16.4)$ & $28(12.8)$ & & $34(15.6)$ & $41(19.1)$ & & $41(18.1)$ & \\
\hline$\geq 7.11$ & $609(69.4)$ & $165(75.7)$ & & $157(72.0)$ & $139(64.7)$ & & $148(65.2)$ & \\
\hline No information & $50(5.7)$ & $13(6.0)$ & & $8(3.7)$ & $11(5.1)$ & & $18(7.9)$ & \\
\hline Birth asphyxia & $191(21.8)$ & $32(14.7)$ & 0.103 & $45(20.6)$ & $50(23.3)$ & 0.511 & $64(28.2)$ & 0.064 \\
\hline Admission to neonatal unit & 349 (39.7) & $69(31.7)$ & 0.678 & $65(29.8)$ & 75 (34.9) & 0.260 & $140(61.7)$ & $<0.001$ \\
\hline
\end{tabular}

Data are presented as variables and percentage or mean \pm standard deviation.

Bold values indicate statistical significance $p<0.05$.

$S D$ standard deviation, $S G A$ small for gestational age, $L G A$ large for gestational age, us-EPO umbilical cord serum erythropoietin.

${ }^{\mathrm{a}}$ Test for relative proportions, Chi-square test or $t$-test. Reference group is us-EPO 20-39. 
Table 2. Adverse outcome according to us-EPO categories.

\begin{tabular}{|c|c|c|c|c|c|c|c|c|}
\hline \multirow[b]{3}{*}{ Outcome } & \multirow{3}{*}{$\begin{array}{l}\text { Total } \\
\text { n (\%) }\end{array}$} & \multicolumn{7}{|c|}{ us-EPO level at birth } \\
\hline & & \multicolumn{2}{|l|}{ Low $<20$} & \multirow{2}{*}{$\begin{array}{l}\text { Average 20-39 } \\
n(\%) \\
\text { Reference group }\end{array}$} & \multicolumn{2}{|c|}{ Elevated 40-100 } & \multicolumn{2}{|l|}{ High $>100$} \\
\hline & & $n(\%)$ & $p$-value ${ }^{a}$ & & $n(\%)$ & $p$-value ${ }^{a}$ & $n(\%)$ & $p$-value ${ }^{a}$ \\
\hline Total & $878(100.0)$ & $218(100.0)$ & & $218(100.0)$ & $215(100.0)$ & & $227(100.0)$ & \\
\hline \multicolumn{9}{|l|}{ Infant mortality } \\
\hline Died at the age of $0-364$ days & $12(1.4)$ & $3(1.4)$ & 0.653 & $2(0.9)$ & $3(1.4)$ & 0.642 & $4(1.8)$ & 0.440 \\
\hline Any major impairment & $26(3.0)$ & $9(4.1)$ & 0.033 & $2(0.9)$ & $5(2.3)$ & 0.245 & $10(4.4)$ & 0.023 \\
\hline Cerebral palsy & $6(0.7)$ & $2(0.9)$ & 0.562 & $1(0.5)$ & $0(0.0)$ & 0.320 & $3(1.3)$ & 0.335 \\
\hline Epilepsia & $5(0.6)$ & $1(0.5)$ & 1.000 & $1(0.5)$ & $2(0.9)$ & 0.554 & $1(0.4)$ & 0.977 \\
\hline Intellectual disability & $7(0.8)$ & $2(0.9)$ & 0.156 & $0(0.0)$ & $3(1.4)$ & 0.080 & $2(0.9)$ & 0.165 \\
\hline Autism spectrum disorder & $4(0.5)$ & $0(0.0)$ & NA & $0(0.0)$ & $1(0.5)$ & 0.313 & $3(1.3)$ & 0.089 \\
\hline $\begin{array}{l}\text { Specific developmental } \\
\text { disorders of speech and } \\
\text { language }\end{array}$ & $18(2.1)$ & $8(3.7)$ & 0.055 & $2(0.9)$ & $6(2.8)$ & 0.148 & $2(0.9)$ & 0.968 \\
\hline $\begin{array}{l}\text { Specific developmental } \\
\text { disorder of motor function }\end{array}$ & 14 (1.6) & $2(0.9)$ & 1.000 & $2(0.9)$ & $4(1.9)$ & 0.401 & $6(2.6)$ & 0.171 \\
\hline $\begin{array}{l}\text { Mixed specific } \\
\text { developmental disorder }\end{array}$ & $8(0.9)$ & $2(0.9)$ & 0.653 & $3(1.4)$ & $3(1.4)$ & 0.986 & $0(0.0)$ & 0.076 \\
\hline $\begin{array}{l}\text { Attention-deficit } \\
\text { hyperactivity disorders }\end{array}$ & $2(0.2)$ & $0(0.0)$ & 0.317 & $1(0.5)$ & $1(0.5)$ & 0.992 & $0(0.0)$ & 0.307 \\
\hline Delayed milestone in childhood & $20(2.3)$ & $4(1.8)$ & 0.522 & $6(2.8)$ & $4(1.9)$ & 0.537 & $6(2.6)$ & 0.943 \\
\hline
\end{tabular}

Bold values indicate statistical significance $p<0.05$.

$N A$ not applicable, us-EPO umbilical cord serum erythropoietin.

${ }^{\mathrm{a}}$ Test for relative propotions. Reference group is us-EPO 20-39.

umbilical cord blood at birth $(n=959)$. Umbilical blood samples are routinely collected from every newborn immediately after birth. During the study period, us-EPO levels were determined systematically as a standard of care of newborns with suspected asphyxia $(n=331)$ based on a 1-min Apgar score of $<4$. In addition, with the separate consent of the parent, a determination was made from samples of newborns with Apgar score $>3$.

The baseline characteristics of these children were collected from the Finnish Medical Birth Register (MBR). ${ }^{15}$ We excluded $2(0.2 \%)$ stillbirths and $79(8.2 \%)$ children with major congenital anomaly defined in the Register on Congenital Malformations. Data of the 878 children that met the inclusion criteria were linked to the Finnish Hospital Discharge Register $(\mathrm{HDR})^{16}$ and the Register on Visual Impairments. ${ }^{17}$

All registers are maintained by the Finnish Institute for Health and Welfare (THL). The MBR contains information on the mother's health and interventions during pregnancy and delivery and the newborn infant's care and outcome during the first week of life. This register contains data on all live births and stillbirths from gestational age of $22+0$ weeks onwards or with a birth weight of at least $500 \mathrm{~g}$. Data provided by the birth hospitals are completed by basic information from the Central Population Register and the Cause of Death Register maintained by Statistics Finland. The HDR records information on admission and discharge dates, main and secondary diagnoses, and surgical procedures for all inpatient care and public hospital outpatient care, covering over $95 \%$ of discharges in Finland. ${ }^{16}$ We used these data to complete information on the mothers' pregnancy-related and delivery-related diagnoses and children's diagnoses. Diagnoses of visual impairments were confirmed from the Register on Visual Impairments, which maintains a record of the prevalence and care of visual impairments.

\section{Classification}

The study population was divided into subgroups according to the us-EPO levels. The us-EPO categories were low (us-EPO $<20 \mathrm{IU} / \mathrm{l}, n=218$ ), average (us-EPO 20-39 IU/l, $n=218$ ), elevated (us-EPO 40-100 IU/l, $n=215$ ), and high (us-EPO $>100 \mathrm{IU} / \mathrm{l}, n=227$ ). We chose us-EPO 20-39 IU/I as the reference group based on studies showing the mean or median fetal EPO level to be within this range at the end of uncomplicated term pregnancies. ${ }^{18-21}$

Apgar scores were provided by midwives or pediatricians according to standardized procedures. The 1- and 5-min Apgar scores were classified into the following three groups: low (0-3), intermediate (4-6), and normal $(7-10){ }^{22}$ In 875 (99.7\%) births, data were available for the 1-min score. For 236 (26.9\%) births, data were missing for the 5-min Apgar score. A common practice in maternal hospitals of the Helsinki University Hospital is that the 5-min score is not recorded if the 1-min score is high (>7) and the child remains in good condition. If the 1-min Apgar score was normal (7-10), the 5-min Apgar score was substituted by the score at $1 \mathrm{~min}$. Newborns were divided into the following subgroups by gestational weeks: early preterm $(\leq 31+6)$ late preterm $(32+0-36+6)$, term $(37+$ $0-41+6)$, and post-term ( $\geq 42+0)$. Gestational age was determined by the first-trimester ultrasound screening, as is routine practice in Finland.

\section{Outcome diagnoses}

Children were followed until the end of 2018, when they were 2-6.5 years old. Neurodevelopmental impairment was recorded if at the end of 2018 the child was recorded in the HDR with the International Statistical Classification of Diseases and Related Health Problems, 10th Revision (ICD-10) codes for one or more of the following major or minor diagnoses. Major morbidity included cerebral palsy (G80.0-G80.9), epilepsy (G40.0-G40.9), intellectual disability (F70.0-F73.9 and F78.0-F79.9), autism spectrum disorder (F84.0-F84.9), and sensorineural defects consisting of visual impairment ( $\mathrm{H} 53.0-\mathrm{H} 53.4$ and $\mathrm{H} 54.0-\mathrm{H} 54.7)$ and hearing impairment (H90.0-H90.8). Minor morbidity included developmental disorders consisting of specific developmental disorders of speech and language (F80.1-80.9), a specific developmental disorder of motor function (F82), mixed specific developmental disorder (F83), and hyperkinetic disorders 
(F90.0-F90.9). Children with delayed development without a more specific diagnosis yet (R62.0) were analyzed separately. Data on birth asphyxia (P21.0-P21.9) are presented in Table 1.

In Finland, all preschool children are invited, and practically all children (99.6\%) attend, to an annual medical and developmental check-up free of charge. ${ }^{23}$ If in these check-ups child's development raises concerns, the child will be referred to the pediatric neurology units of either secondary or tertiary care public hospitals for a more detailed assessment. A specialist sets diagnoses based on medical history, clinical multidisciplinary evaluation, brain imaging, and clinical neurophysiological investigations when appropriate. The most severe neurodevelopmental disorders (cerebral palsy, severe intellectual disability, deafness, and blindness) usually occur and are accordingly diagnosed within the first 2 years of life. Epilepsy is diagnosed at the time of the first symptoms, and intellectual disability and autism spectrum disorder are mostly diagnosed at the latest by the beginning of school at 6 years of age. The diagnosis "delayed milestone in childhood" is set when the child's development is not ageappropriate, but a more accurate diagnosis cannot yet be made. Diagnoses are included in the HDR as soon as they are established. Data on deaths were available in the MBR only for the first year of life.

\section{Ethics}

For this study, the THL, the hospital district of Helsinki and Uusimaa, and the Ethics Committee for gynecology and obstetrics, pediatrics, and psychiatry of Helsinki University Hospital provided the authorizations (THL/ 317/5.05.00/2019; \$12/28.2.2018/HUS/185/2018; \$181/24.8.2017/HUS/ 2313/2017) required by the data protection legislation in Finland. All register and hospital data linkages were performed using unique personal identification numbers anonymized by the authorities.

\section{Statistical analysis}

Characteristics of newborns and their mothers are shown as a number of values with percentages for categorical variables and as means with standard deviations for normally distributed continuous variables. us-EPO level categories were compared with each other by test for relative proportions, chi-square test, or t-test when appropriate (Tables 1 and 2).

Association of us-EPO levels with neurodevelopmental morbidity and infant mortality were analyzed by logistic regression using multivariate enter models adjusted for gestational age with term group as a reference (Table 3). The results are shown as odds ratios (OR) with $95 \%$ confidence intervals $(\mathrm{Cl})$. A comparison of the distribution of us-EPO levels was made by using Kolmogorov-Smirnov two-samples test (Fig. 2). Correlation analysis was performed by Pearson correlation coefficients.

Statistical analyses were performed with IBM SPSS Statistics version 20.0 (2014) and SAS version 9.4 (SAS Institute Inc., Cary, North Carolina). $p$ values lower than 0.05 were considered statistically significant.

\section{RESULTS}

\section{Clinical characteristics}

Of a total of 878 live-born children, $11.4 \%(n=100)$ were born preterm, $11.6 \%(n=102)$ post-term, and 3.8\% $(n=33)$ from multiple pregnancies. The mean gestational age was 39.6 (SD 2.8) weeks. The study population included 866 mothers with a mean age of 31.7 (SD 5.3) years. Maternal gestational diabetes incidence was $15.0 \%$, diabetes type I $3.0 \%$, hypertension $3.0 \%$, and preeclampsia $4.1 \%$. Birth asphyxia was diagnosed in $21.8 \%(n=191)$. Characteristics of newborns are shown in Table 1. Maternal characteristics are presented in the supplement.

\section{Erythropoietin}

The median us-EPO level was 40.8 IU/I (interquartile range 20.3-104.0 IU/I). In the study population, there were 227 (25.9\%) children with high us-EPO levels (>100 IU/I). Of these children, 81 (9.2\%) had us-EPO levels $>500 \mathrm{IU} / \mathrm{I}$ and 54 (6.2\%) children $>1000$ IU/I. us-EPO levels <10 IU/I were observed in 66 (7.5\%) children.

Children with high us-EPO levels were more likely to be born by cesarean section $(64.3 \%$ vs. $25.7 \%, p<0.001)$, to have a lower mean birth weight ( 3307 vs. $3504 \mathrm{~g}, p=0.001$ ), and have an Apgar score $<7$ at $1(76.2 \%$ vs. $52.3 \%, p<0.001)$ and $5 \mathrm{~min}(46.7 \%$ vs. $25.7 \%, p<0.001)$ than children with us-EPO 20-39 IU/l. These 
100,000

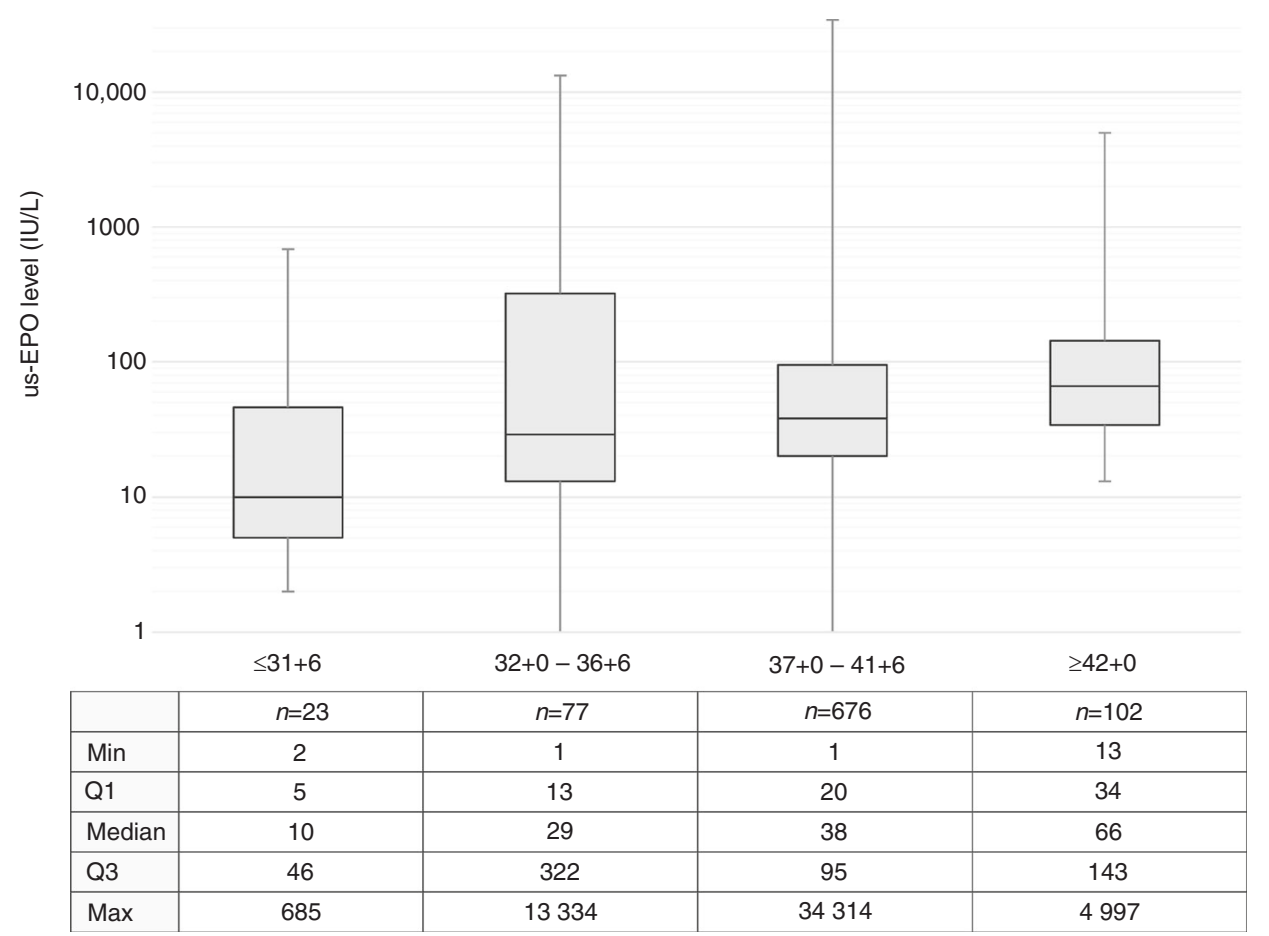

Fig. 1 Distribution of umbilical cord serum erythropoietin (us-EPO) levels in different gestational age groups. us-EPO levels are plotted on a logarithmic scale and data on group size and quartiles of us-EPO in each group are shown in the table below the graph.

children were also more likely to be admitted to the neonatal unit than children with us-EPO $20-39$ IU/I $(61.7 \%$ vs. $29.8 \%, p<0.001)$. Children with low us-EPO levels were more likely to be born preterm $(19.3 \%$ vs. $7.3 \%, p<0.001)$, less likely to be born post-term (3.2\% vs. $10.6 \%, p=0.002)$, and more likely had a lower mean birth weight (3224 vs. $3504 \mathrm{~g}, p<0.001)$ than children with us-EPO 20-39 IU/I (Table 1).

Us-EPO levels correlated with gestational age $(r=0.526)$. The median us-EPO level increased by gestational age (Fig. 1).

\section{Neurodevelopmental morbidity and mortality}

At the end of 2018 , of a total of 878 children, $18.1 \%(n=159)$ were under 3 years of age, $16.5 \%(n=145)$ were 3 years of age, $12.2 \%$ $(n=107)$ were 4 years of age, $38.2 \%(n=335)$ were 5 years of age, and $13.7 \%(n=120)$ were 6 years of age. After combining the usEPO data with registers, 82 (9.3\%) children were identified with any neurodevelopmental diagnosis. At least one major neurodevelopmental impairment was identified in $26(3.0 \%)$ children, of whom 1 (3.8\%) child was born early preterm, 4 (15.4\%) children were born late preterm, 19 (73.1\%) children were born term, and 2 (7.7\%) post-term. Of these 26 children, 4 children had two major diagnoses and 1 child had four major diagnoses. The most common severe neurodevelopmental impairment in the study population was sensorineural defects $(n=11,1.3 \%)$, consisting of $7(0.8 \%)$ children with visual impairment and $4(0.5 \%)$ children with hearing impairment. No child had both visual and hearing impairment. In addition, 36 (4.1\%) children had at least one minor neurodevelopmental disorder. In the other 20 (2.3\%) children, development was delayed without an as-yet more specific diagnosis. (Table 2)

A total of 12 (1.4\%) live-born children died; most of them (75.0\%) died during the perinatal period. Three newborn infants died during the first day of life, 6 died at the age of 1-6 days, 1 child died later during the first month, and 2 died later in the first year of life.

\section{Results from analyses}

A severe neurodevelopmental impairment was more likely to be diagnosed in children with an abnormal us-EPO, whether low $(4.1 \%$ vs. $0.9 \%, p=0.033)$ or high $(4.4 \%$ vs. $0.9 \%, p=0.023)$, than in children with us-EPO 20-39 IU/l (Table 2). High us-EPO levels $(>100 \mathrm{IU} / \mathrm{l})$ at birth were associated with an increased risk of severe neurodevelopmental morbidity (OR 4.87, $95 \% \mathrm{Cl}$ 1.05-22.58) in the model adjusted for the gestational age. No association of high us-EPO with minor neurodevelopmental disorders or with infant mortality was observed (Table 3). No statistically significant difference in the distribution of us-EPO levels was found between children with or without any neurodevelopmental diagnosis (Fig. 2).

\section{DISCUSSION}

In this study, we determined whether endogenous us-EPO is a useful biomarker to assess and predict the risk of neurodevelopmental morbidity. Our hypothesis was that not only severe neurodevelopmental diseases but also minor neurodevelopmental disorders and mortality occur more often in children with high us-EPO than children with average us-EPO.

We observed that high us-EPO concentration at birth, regardless of gestational age, was associated with an increased risk of major neurodevelopmental morbidity in early childhood. No statistically significant association occurred between us-EPO and minor neurodevelopmental disorders. High us-EPO was not associated with infant mortality. We observed that the us-EPO level at birth was dependent on gestational age; the lower the gestational age, the lower the median us-EPO level. 
100,000

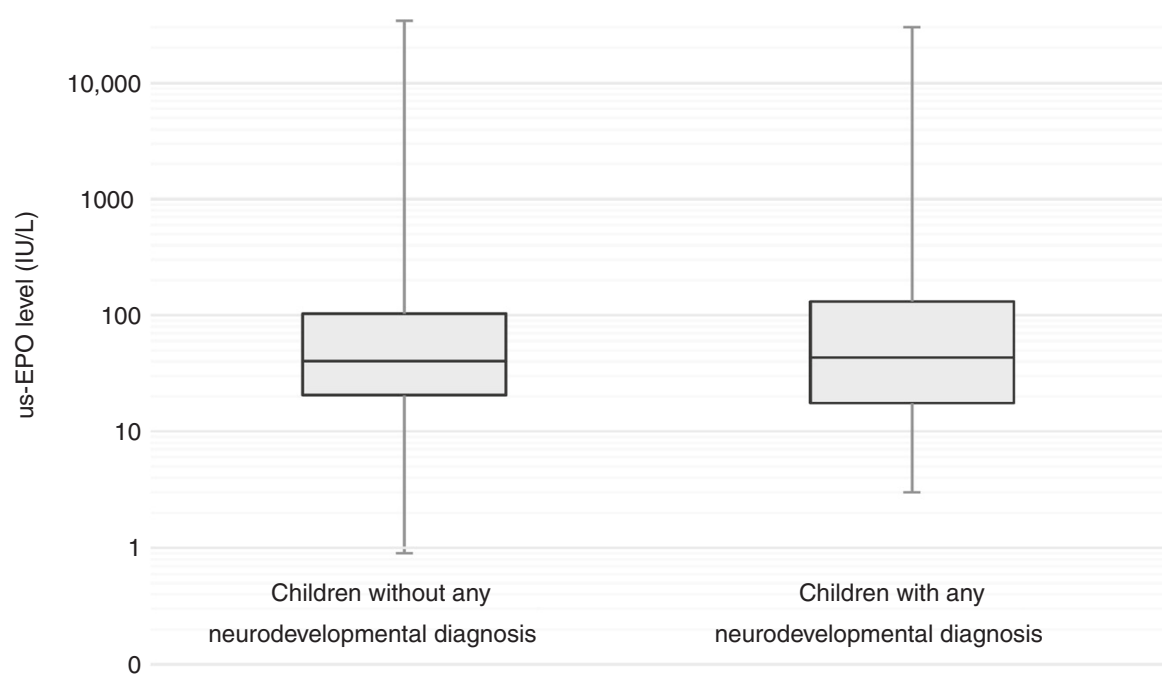

\begin{tabular}{|l|c|c|c|}
\hline & $n=784$ & $n=82$ & $p$-value ${ }^{\mathrm{a}}$ \\
\hline Min & 1 & 3 & \multirow{2}{*}{0.272} \\
\hline Q1 & 21 & 43 & \\
\hline Median & 40 & 132 & \\
\hline Q3 & 103 & 29970 & \\
\hline Max & 34314 & & \\
\hline
\end{tabular}

Fig. 2 Distribution of umbilical cord serum erythropoietin (us-EPO) levels at birth in children without and with any later neurodevelopmental diagnosis. Neurodevelopmental morbidity includes cerebral palsy, epilepsy, intellectual disability, autism spectrum disorders, sensorineural defects, and minor neurodevelopmental disorders. Infant deaths in the first year $(n=12)$ are excluded. us-EPO levels are plotted on a logarithmic scale and data on group size and quartiles of us-EPO in both groups are shown in the table below the graph. A comparison of the distribution of us-EPO levels between groups was made by using. ${ }^{a}$ Kolmogorov-Smirnov two-sample test.

\section{Comparison with previous findings}

To our knowledge, only two studies have previously assessed the significance of high endogenous EPO levels of newborns in subsequent neurodevelopmental morbidity. One follow-up study from 1988 revealed that high umbilical plasma EPO levels after acute birth asphyxia ( $n=31$, of whom 5 preterm children), but not after pre-eclampsia ( $n=62$, of whom 42 preterm children), were associated with an increased risk of cerebral palsy, a Bayley Mental Developmental Index below 90 , or death at the 2 years of age. ${ }^{13}$ Our results are rather similar to these preliminary results obtained with a small study population, although our analyzes were not limited to the asphyxia group alone. Korzeniewski et al. determined endogenous EPO levels on day 14 from 786 infants born before the 28th week of gestation. The highest quarter of endogenous EPO levels were associated with very low $(<55)$ Mental (OR 2.3; 95\% Cl 1.5-3.5) or Psychomotor (OR 2.4; $95 \% \mathrm{Cl}$ 1.6-3.7) Development Indices (or both) at 2 years of age. ${ }^{14}$ In our study, blood samples were collected immediately after birth in order to more accurately assess the significance of us-EPO concentration in relation to oxygen deficiency at birth. Our cohort also consisted mostly of children (88.6\%) born term or post-term. In contrast to these two previous studies, we assessed the association of us-EPO with precisely defined diagnoses, and at the time of evaluation, most children (51.9\%) were over 5 years of age.

In addition, Forestier et al. reported that fetal EPO levels remained stable throughout pregnancy with no correlation between EPO and gestational age, when EPO levels were measured in 163 fetal intrauterine blood samples from the 18th week of gestation to the end of pregnancy. ${ }^{21}$ Subsequently, Seikku et al. found us-EPO to correlate with gestational age among vaginal term and post-term births $(r=0.250){ }^{24}$ while Bahr et al. reported no difference in us-EPO levels between term $(n=13)$ and preterm $(n=10){ }^{25}$ Our results confirmed gestational agedependent correlation in EPO levels, when EPO was determined from umbilical cord serum samples at birth.

\section{Deliberation}

Hypoxia causes brain damage. Since EPO levels increase due to hypoxia, EPO could be a useful biomarker to predict later neurodevelopmental problems. On the other hand, several in vitro and in vivo studies have shown the effects of EPO to be anti-apoptotic, anti-inflammatory, and antioxidative., ${ }^{4,26-28}$ EPO also promotes brain revascularization and neurogenesis. ${ }^{4,5,26-28}$ Preliminary results in children suggest that EPO is also involved in the regulation of systemic inflammation and may thereby contribute to subsequent events. Levels of EPO in blood on postnatal days 1,7 , and 14 vary monotonically with inflammationrelated proteins in extremely preterm children. ${ }^{29}$ High EPO combined with high cytokine concentrations in the blood sample taken during the first postnatal month has been associated with an increased risk of ADHD symptoms in 10-year-old children born extremely preterm. ${ }^{30}$ Zareen et al. assessed school-age children 
with a background of neonatal encephalopathy (NE) and agematched controls for their cytokine responses and neurodevelopmental outcome. ${ }^{31}$ They demonstrated that EPO levels in post-NE children were higher compared with controls, especially in response to in vitro stimulation with lipopolysaccharide.

The evidence of a neuroprotective role of EPO is controversial: recent randomized, placebo-controlled trials have shown no benefit of high-dose EPO treatment in improving neurological outcome. $^{8-10}$ Our finding of the association of high us-EPO levels with severe neurodevelopmental morbidity may indicate that, despite the neurotrophic effects of EPO, a significant portion of children still develop brain damage when hypoxia has been intense enough to increase us-EPO to a very high level. In our study, a fairly large number of asphyxia diagnoses (22\%) probably reflects the inclusion criteria of low Apgar scores in selecting the cohort.

Another consideration is timing. It has previously been reported that plasma EPO levels do not start to increase until 90 min after the beginning of acute hypoxia in human adults ${ }^{32}$ and within $2-3 \mathrm{~h}$ in fetuses in animal experiments. ${ }^{33,34}$ The peak values of EPO levels are reached in 1-2 days and fall thereafter. ${ }^{1,35}$ EPO is not stored in tissues and the half-life of EPO in the blood is $2-4 \mathrm{~h}$ in newborn infants. ${ }^{36}$ Thus, even if the newborn infant suffers from the lack of oxygen during childbirth, if the delivery is rapid, us-EPO levels may not yet show the entire magnitude of increase at the time of birth. In addition, umbilical cord blood has been shown to differ significantly from the subsequent postnatal blood sample, inter alia, in cell composition, plasma protein concentrations, and immune cell phenotypes. ${ }^{37} \mathrm{~A}$ second postnatal sample could thus be beneficial and substantive in the timing of hypoxia and in evaluating the significance of EPO as a marker of birth asphyxia.

When interpreting the us-EPO level in clinical use, gestational age should be considered, because it appears that birth itself, or associated with hypoxia, may not increase the us-EPO level in premature children. Overall, the role of endogenous us-EPO to predict the risk of neurodevelopmental morbidity in clinical use is likely to be minor and without careful evaluation may even lead to misinterpretation and misdirect the child's follow up.

\section{Limitations and strengths}

Due to the standard practice of collecting umbilical blood samples immediately after birth, we used 1-min Apgar scores to identify newborns to enroll in the study, although 5-min scores are known to better predict long-term outcomes. ${ }^{38,39}$ The notable limitation of our study was that $27 \%$ of the 5 -min Apgar scores were unrecorded due to the common practice of not providing a 5-min score for children with a high 1-min score. Based on clinical experience, there was no medical need to record 5-min scores in these cases.

In addition, we had no data on obstetric and neonatal complications, such as previous transient fetal bleeding or erythema infectiosum infection, or durations of deliveries that could affect us-EPO levels. Data on neonatal interventions, which may reduce the risk of death and adverse neurodevelopmental outcome, such as induced hypothermia for children with hypoxic-ischemic encephalopathy, were also not available. It should also be noted that only severe impairments can be diagnosed during the first few years of life. Hyperkinetic disorders, specific developmental disorders, and mild intellectual disability can often only be detected later. Nevertheless, the strength of this study was a study population of good size that was not limited to risk groups. Thus, the results are better generalizable to the population level. The data were collected from high-quality and comprehensive registers ${ }^{15-17}$ and diagnoses were specifically defined and always made by a specialist in neurodevelopmental disorders. The study design allowed for the clinical relevance of endogenous us-EPO to be assessed.

\section{CONCLUSION}

us-EPO levels were dependent on gestational age and were lower in premature children, which should be considered when interpreting the us-EPO level. Although high us-EPO concentration at birth was associated with an increased risk of neurodevelopmental morbidity in early childhood regardless of gestational age, determining endogenous us-EPO appears to be of minor clinical utility.

\section{REFERENCES}

1. Jelkmann, W. Regulation of erythropoietin production. J. Physiol. 589, 1251-1258 (2011).

2. Teramo, K. A. \& Widness, J. A. Increased fetal plasma and amniotic fluid erythropoietin concentrations: markers of intrauterine hypoxia. Neonatology $\mathbf{9 5}$, 105-116 (2009).

3. Widness, J. A., Schmidt, R. L. \& Sawyer, S. T. Erythropoietin transplacental passage -review of animal studies. J. Perinat. Med. 23, 61-70 (1995).

4. Rangarajan, V. \& Juul, S. E. Erythropoietin: emerging role of erythropoietin in neonatal neuroprotection. Pediatr. Neurol. 51, 481-488 (2014).

5. Martini, S., Austin, T., Aceti, A., Faldella, G. \& Corvaglia, L. Free radicals and neonatal encephalopathy: mechanisms of injury, biomarkers, and antioxidant treatment perspectives. Pediatr. Res. 87, 823-833 (2020).

6. Ohls, R. K. et al. Preschool assessment of preterm infants treated with darbepoetin and erythropoietin. Pediatrics 137, e20153859 (2016).

7. Fischer, H. S., Reibel, N. J., Buhrer, C. \& Dame, C. Prophylactic early erythropoietin for neuroprotection in preterm infants: a meta-analysis. Pediatrics 139, e20164317 (2017).

8. Natalucci, G. et al. Effect of early prophylactic high-dose recombinant human erythropoietin in very preterm infants on neurodevelopmental outcome at 2 years: a randomized clinical trial. JAMA 315, 2079-2085 (2016).

9. Juul, S. E. et al. A randomized trial of erythropoietin for neuroprotection in preterm infants. N. Engl. J. Med. 382, 233-243 (2020).

10. Natalucci, G. et al. Neurodevelopmental outcomes at age 5 years after prophylactic early high-dose recombinant human erythropoietin for neuroprotection in very preterm infants. JAMA 324, 2324-2327 (2020).

11. Holm, M. et al. Systemic endogenous erythropoietin and associated disorders in extremely preterm newborns. Arch. Dis. Child. Fetal Neonatal Ed. 101, 458-463 (2016).

12. Sweetman, D. U., Onwuneme, C., Watson, W. R., Murphy, J. F. \& Molloy, E. J. Perinatal asphyxia and erythropoietin and VEGF: serial serum and cerebrospinal fluid responses. Neonatology 111, 253-259 (2017).

13. Ruth, V., Autti-Ramo, I., Granstrom, M. L., Korkman, M. \& Raivio, K. O. Prediction of perinatal brain damage by cord plasma vasopressin, erythropoietin, and hypoxanthine values. J. Pediatr. 113, 880-885 (1988).

14. Korzeniewski, S. J. et al. Elevated endogenous erythropoietin concentrations are associated with increased risk of brain damage in extremely preterm neonates. PLOS ONE 10, e0115083 (2015).

15. Gissler, M. \& Shelley, J. Quality of data on subsequent events in a routine Medical Birth Register. Med. Inform. Internet Med. 27, 33-38 (2002).

16. Sund, R. Quality of the Finnish Hospital Discharge Register: a systematic review. Scand. J. Public Health 40, 505-515 (2012).

17. Pukkala, E., Verkasalo, P. K., Ojamo, M. \& Rudanko, S. L. Visual impairment and cancer: a population-based cohort study in Finland. Cancer Causes Control 10, 13-20 (1999).

18. Widness, J. A. et al. Increased erythropoiesis and elevated erythropoietin in infants born to diabetic mothers and in hyperinsulinemic rhesus fetuses. J. Clin. Invest. 67, 637-642 (1981).

19. Teramo, K. A. et al. Amniotic fluid erythropoietin correlates with umbilical plasma erythropoietin in normal and abnormal pregnancy. Obstet. Gynecol. 69, 710-716 (1987).

20. Eckardt, K. U. et al. Serum immunoreactive erythropoietin of children in health and disease. Eur. J. Pediatr. 149, 459-464 (1990).

21. Forestier, F., Daffos, F., Catherine, N., Renard, M. \& Andreux, J. P. Developmental hematopoiesis in normal human fetal blood. Blood 77, 2360-2363 (1991).

22. American Academy of Pediatrics Committee on Fetus and Newborn, American College of Obstetricians and Gynecologists Committee on Obstetric Practise. The Apgar score. Pediatrics 136, 819-822 (2015).

23. The Finnish Institute for Health and Welfare (THL). Lapset, nuoret ja perheet: Lastenneuvola. https://thl.fi/fi/web/lapset-nuoret-ja-perheet/sote-palvelut/aitiysja-lastenneuvola/lastenneuvola. Accessed 23 Apr 2021.

24. Seikku, L. et al. Amniotic fluid and umbilical cord serum erythropoietin in term and prolonged pregnancies. Eur. J. Obstet. Gynecol. Reprod. Biol. 233, 1-5 (2019).

25. Bahr, T. M. et al. Is the erythropoietin-erythroferrone-hepcidin axis intact in human neonates? Blood Cells Mol. Dis. 88, 102536 (2021). 
26. Juul, S. E. \& Pet, G. C. Erythropoietin and neonatal neuroprotection. Clin. Perinatol. 42, 469-481 (2015)

27. Teramo, K. A., Klemetti, M. M. \& Widness, J. A. Robust increases in erythropoietin production by the hypoxic fetus is a response to protect the brain and other vital organs. Pediatr. Res. 84, 807-812 (2018).

28. Suresh, S., Rajvanshi, P. K. \& Noguchi, C. T. The many facets of erythropoietin physiologic and metabolic response. Front. Physiol. 10, 1534 (2020).

29. Logan, J. W. et al. Endogenous erythropoietin varies significantly with inflammationrelated proteins in extremely premature newborns. Cytokine 69, 22-28 (2014).

30. Allred, E. N. et al. Systemic inflammation during the first postnatal month and the risk of attention deficit hyperactivity disorder characteristics among 10 year-old children born extremely preterm. J. Neuroimmune Pharmacol. 12, 531-543 (2017).

31. Zareen, Z. et al. Cytokine dysregulation persists in childhood post neonatal encephalopathy. BMC Neurol. 20, 115 (2020).

32. Eckardt, K. U. et al. Rate of erythropoietin formation in humans in response to acute hypobaric hypoxia. J. Appl. Physiol. 66, 1785-1788 (1989).

33. Widness, J. A. et al. Temporal response of immunoreactive erythropoietin to acute hypoxemia in fetal sheep. Pediatr. Res. 20, 15-19 (1986).

34. Kakuya, F., Shirai, M., Takase, M., Ishii, N. \& Okuno, A. Effect of hypoxia on amniotic fluid erythropoietin levels in fetal rats. Biol. Neonate 72, 118-124 (1997).

35. Abbrecht, P. H. \& Littell, J. K. Plasma erythropoietin in men and mice during acclimatization to different altitudes. J. Appl. Physiol. 32, 54-58 (1972).

36. Widness, J. A. et al. Erythropoietin pharmacokinetics in premature infants: developmental, nonlinearity, and treatment effects. J. Appl. Physiol. 80, 140-148 (1996).

37. Olin, A. et al. Stereotypic immune system development in newborn children. Cell 174, 1277-1292 (2018).

38. Casey, B. M., McIntire, D. D. \& Leveno, K. J. The continuing value of the Apgar score for the assessment of newborn infants. N. Engl. J. Med. 344, 467-471 (2001).

39. Leinonen, E. et al. Low Apgar scores at both one and five minutes are associated with long-term neurological morbidity. Acta Paediatr. 107, 942-951 (2018).

\section{ACKNOWLEDGEMENTS}

The study was supported by The Foundation for Pediatric Research, the Arvo and Lea Ylppö Foundation, the Päivikki and Sakari Sohlberg Foundation, and the Finnish Medical Foundation.

\section{AUTHOR CONTRIBUTIONS}

All authors have made substantial contributions to concept and design, acquisition of data, or analysis and interpretation of data. E.R. drafted the initial manuscript and all other authors revised it critically for important intellectual content. All authors have approved the final version to be published.

\section{FUNDING}

Open access funding provided by University of Helsinki including Helsinki University Central Hospital.

\section{COMPETING INTERESTS}

The authors declare no competing interests.

\section{CONSENT STATEMENT}

Patient consent was not required.

\section{ADDITIONAL INFORMATION}

Supplementary information The online version contains supplementary material available at https://doi.org/10.1038/s41390-021-01679-0.

Correspondence and requests for materials should be addressed to E.J.R.

Reprints and permission information is available at http://www.nature.com/ reprints

Publisher's note Springer Nature remains neutral with regard to jurisdictional claims in published maps and institutional affiliations.

(i) Open Access This article is licensed under a Creative Commons Attribution 4.0 International License, which permits use, sharing, adaptation, distribution and reproduction in any medium or format, as long as you give appropriate credit to the original author(s) and the source, provide a link to the Creative Commons license, and indicate if changes were made. The images or other third party material in this article are included in the article's Creative Commons license, unless indicated otherwise in a credit line to the material. If material is not included in the article's Creative Commons license and your intended use is not permitted by statutory regulation or exceeds the permitted use, you will need to obtain permission directly from the copyright holder. To view a copy of this license, visit http://creativecommons. org/licenses/by/4.0/.

(c) The Author(s) 2021 